\title{
Open submucosal tunneling endoscopic resection for special submucosal tumors originating from the muscularis propria
}

\section{Silin Huang}

Shenzhen Hospital of Southern Medical University

\section{Sufang Tu}

Shenzhen Hospital of Southern Medical University

Jingwen Fu

Southern Medical University Nanfang Hospital

Xiaowei Tang

Affiliated Hospital of Southwest Medical University

Genhua Yang

Shenzhen Hospital of Southern Medical University

Wei Gong ( $\nabla$ drgwei@foxmail.com )

https://orcid.org/0000-0003-2498-7615

\section{Research article}

Keywords: esophageal submucosal tumors, submucosal tunneling endoscopic resection, open submucosal tunneling endoscopic resection, efficacy, safety

Posted Date: April 12th, 2020

DOl: https://doi.org/10.21203/rs.3.rs-21242/v1

License: (c) (i) This work is licensed under a Creative Commons Attribution 4.0 International License. Read Full License 


\section{Abstract}

Background and Purpose Esophageal submucosal tumors (SMTs) are primarily benign but can have the potential for malignancy. Surgery is the primary treatment; however, the development of endoscopic techniques has promoted the use of endoscopic resection for esophageal SMTs. Submucosal tunneling endoscopic resection (STER) is the current optimal treatment for esophageal SMTs; however, it is challenging to perform in cases of tumors located in the upper esophagus and cardia as well as those with a diameter $>3.5 \mathrm{~cm}$. Based on STER, we proposed and used open STER (O-STER) for special esophageal SMTs. The study aimed to evaluate the safety and efficacy of O-STER for special esophageal SMTs, including those located in the upper esophagus and cardia and those with a diameter $>3.5 \mathrm{~cm}$.

Methods The study enrolled 21 patients whose baseline characteristics, clinical data, and follow-up results were retrospectively analyzed.

Results A total of 21 lesions were resected. The mean patient age was $44.2 \pm 14.4$ years. The mean tumor size was $38.2 \pm 12.2 \mathrm{~mm} ; 16(76.2 \%)$ tumors had a diameter $\geq 3.5 \mathrm{~cm}$. Tumors were in the cardia in 14 $(66.7 \%)$ cases and in the upper esophagus in $2(9.5 \%)$ cases. The mean operation time was $56.7 \pm 19.3$ minutes. The en bloc and complete resection rates were both $100 \%$. There was $1(4.8 \%)$ case of subcutaneous emphysema; no other serious complications were noted. Pathological examinations suggested that most tumors were leiomyomas and had negative margins. During the mean follow-up period of 26.7 months, no cases of residue, recurrence, or metastasis were observed.

Conclusions O-STER is a safe and effective treatment for special esophageal SMTs that can reduce operative difficulty and complication rate.

\section{Introduction}

Submucosal tumors (SMTs) are mesenchymal cell-derived tumors with the mucosa, submucosa, or muscularis propria (MP) as the primary sites that can develop anywhere in the gastrointestinal tract [1, 2]. Esophageal SMT is a relatively rare esophageal disease that accounts for $<1 \%$ of esophageal tumors. Esophageal SMTs usually do not cause clinical symptoms and are often detected incidentally on endoscopic examinations [3, 4]. The majority of SMTs are benign, but some have the potential for malignant transformation. Although imaging or endoscopic ultrasonography can assist in making a preoperative judgment of the nature of the tumor, it cannot be used for accurate characterization. Contrarily, biopsy can damage the tumor and cause its dissemination. Therefore, complete tumor resection to determine its pathological type is essential.

In the past, surgery, generally comprising thoracotomy and video-assisted thoracoscopic enucleation, was the main approach to the resection of esophageal SMTs. However, surgery is associated with severe tissue trauma, a high perforation rate, and slow patient recovery. With the rapid development of endoscopic techniques, endoscopic resection has emerged as a new method of treating esophageal SMTs. Endoscopic submucosal dissection (ESD) has gradually been applied for the resection of SMTs 
originating from the MP; however, it is associated with a low complete resection rate of only $64-75 \%$ [5, 6]. Subsequently developed treatment approaches, including endoscopic submucosal excavation (ESE) and endoscopic full-thickness resection (EFTR), have achieved complete resection rates as high as $90 \%$ [7]. However, due to the dissection of tissues in deeper layers, the incidence of bleeding and perforation is also significantly increased in ESE and EFTR.

In 2010, Inoue et al. [8] first reported on peroral endoscopic myotomy (POEM) for the treatment of achalasia, which led to the development of tunnel endoscopy. In 2012, based on POEM and ESD, Xu first reported submucosal tunneling endoscopic resection (STER) for the treatment of SMTs originating from the MP layer [9]. The advantage of this technique is that the creation of the submucosal tunnel helps preserve the integrity of the intact gastrointestinal mucosa, which can minimize wound damage, expedite wound healing, and further avoid the incidence of gastrointestinal fistula and perforation. Many centers in China and other countries have attempted this tunneling technique. In 2012, our center also employed this technique for the treatment of SMTs in the upper gastrointestinal tract and confirmed its safety and efficacy. It was also reported in the literature that STER has a complete resection rate as high as 70.2$100 \%[9,10]$. The associated complications mainly include gas-related problems and pleural effusions, which can be treated effectively with conservative medical therapy. Thus, STER is currently the optimal method for esophageal SMTs originating from the MP.

Although studies have confirmed that STER is safe and effective for the treatment of esophageal SMTs, most suggest that the maximum diameter of such esophageal SMTs be $\leq 3.5 \mathrm{~cm}$ as it is difficult to perform STER for larger tumors. Further, there is limited operating space in esophageal SMTs in the upper esophagus or the cardia, which increases the level of difficulty. After accumulating abundant experience, our center developed the open submucosal tunneling endoscopic resection (O-STER) technique. With opening of the distal end of the tunnel to form a second tunnel opening, the operating space in the tunnel is increased, enhancing the operational field of view, reducing the pressure inside the tunnel, and making the operation easier for certain esophageal SMTs such as those located in the cardia or upper esophagus or those with a diameter $>3.5 \mathrm{~cm}$. Our center utilized the O-STER technique for the resection of esophageal SMTs in 21 patients. The clinical data of the 21 patients were retrospectively collected, and their baseline characteristics, clinical characteristics, and pathological and follow-up results were statistically analyzed to evaluate the safety and efficacy of O-STER for the treatment of these esophageal SMTs.

\section{Materials And Methods}

1.

Patients

Data from cases of esophageal SMTs treated with O-STER at Shenzhen Hospital and Nanfang Hospital of Southern Medical University between September 2014 and May 2019 were retrospectively analyzed. A total of 21 patients (21 lesions) were included in the study. The inclusion criteria were as follows: (1) 
esophageal SMT detected and confirmed on white light endoscopy; (2) endoscopic ultrasonography indicating esophageal SMT derived from the MP and growing into the lumen; (3) SMT located in the upper esophagus or the cardia or those with a diameter $>3.5 \mathrm{~cm}$; (4) CT examination suggesting a benign tumor; and (5) ability to tolerate anesthesia with tracheal intubation and provision of a signed informed consent.

All patients underwent preoperative electrocardiography (ECG), chest X-ray, routine blood testing, blood biochemistry, and coagulation testing; patients with surgical contraindications were excluded. Preoperative endoscopic ultrasonography and enhanced chest and abdominal CT were necessary to assist in the evaluation of tumor nature and origin as well as the conditions of the surrounding lymph nodes and blood vessels. All patients were informed of the procedure and the aims and received detailed explanations about the treatment and complications. Each provided informed consent prior to participating.

Food and water were prohibited in all patients for at least 6 hours before the operation. Intraoperatively, each patient was placed in the left lateral decubitus position under oxygen inhalation, electrocardiogram monitoring, and general anesthesia with endotracheal intubation. All operations were performed by experienced endoscopists. This study was approved by the Ethics Committee of Shenzhen Hospital of Southern Medical University.

2.

Endoscopic equipment and O-STER procedures

The endoscopic equipment primarily included an endoscope (GIF-Q260J, Olympus, Tokyo, Japan; EG580RD, Fujifilm, Tokyo, Japan), a transparent distal cap (MH-588, Olympus; DH-28GR; Fujifilm), a highfrequency generator (VIO200D, ERBE, Germany), a carbon dioxide (CO2) insufflator (UCR, Olympus, Japan; GW-100, Fujifilm, Tokyo, Japan), and a snare (SD-210U-25, Olympus). The tumor dissection was performed using a flush knife (DK2618JB-20, Fujifilm) or hybrid knife (ERBE, Tübingen, Germany). Hemostatic forceps (Coagrasper, FD-410LR, Olympus) were used to control bleeding, while endoscopic clips (Micro-Tech, Nanjing, China) were used for wound closure.

The O-STER procedures included the following steps: (1) submucosal injection: submucosal injection of $0.2 \%$ indigo carmine and 1:100000 epinephrine in normal saline at a distance of approximately $3-5 \mathrm{~cm}$ proximal to the tumor so that the mucosa was fully lifted; (2) creation of the tunnel entrance: when the mucosa was fully lifted, a 2-cm-long longitudinal mucosal incision was made on the mucosal layer to create the tunnel entrance, followed by the dissection of $\geq 0.5 \mathrm{~cm}$ of the submucosa along the longitudinal incision; (3) creation of the submucosal tunnel: the endoscope was advanced into the submucosa with the aid of the distal attachment, a straight submucosal tunnel was constructed from the oral to the anal side, and the submucosa was gradually dissociated downward against the muscular layer until the tumor was visible; (4) SMT removal: the mucosa at the distal end of the tumor was cut open along the longitudinal axis of the tumor and a second tunnel opening was created. The tumor was gradually dissected along its capsule. During the resection, the tumor was gradually extruded out of the 
tunnel through the second opening. The dissection continued until the tumor was completely dissociated, and the tumor was removed from the body; and (5) tunnel closure: the wound inside the tunnel was carefully inspected and flushed; metal clips were used to suture from the bottom to the top and gradually close the two tunnel openings.(Fig. 1 and Fig. 2 shows the key procedures of O-STER; Fig. 3 and Fig. 4 shows two cases of O-STER for esophageal and cardial SMTs.)

3.

Postoperative management

After the operation, the patients received oxygen and underwent ECG and vital signs monitoring. All patients were required to remain nil per os before resuming their diet and water, and parenteral nutrition was maintained for at least 48 hours. When the patients recovered and their conditions stabilized, their diet was gradually restored to a normal diet within 2 weeks starting with a transitional liquid diet for approximately 3 days. At the same time, prophylactic intravenous antibiotics (second- or third generation cephalosporins) were administered for 3 days. All patients were administered intravenous proton pump inhibitors (PPIs) for 3 consecutive days postoperatively and continued to receive oral PPIs for 4 weeks. The patients were closely observed for postoperative discomfort and abnormal signs and symptoms, such as fever, chest distress, shortness of breath, abdominal pain, abdominal distension, and cyanosis. They were also carefully monitored for complications such as subcutaneous emphysema, pneumothorax, and pleural effusion, and examinations by chest X-ray, erect abdominal X-ray, or chest CT were conducted in a timely manner to prevent postoperative complications and expedite any necessary treatment.

The resected specimens were examined for tumor and capsule intactness, fixed in $10 \%$ formaldehyde solution, and subjected to routine pathological examination. If necessary, immunohistochemical staining was performed. The tested markers included smooth muscle actin(SMA), desmin, S-100, CD117, CD34, DOG-1, and Ki67. For lesions with a confirmed diagnosis of a gastrointestinal stromal tumor (GIST), the risk classification was determined based on tumor size and mitotic index. En bloc resection was defined as the resected tumor having an intact capsule with the tissue excised in a single block. Complete resection was defined as total tumor resection under endoscopy with negative horizontal and vertical margins and no residual tumor tissue under microscopic examination of the pathological sections.

\section{4.}

Follow-up strategy

All patients were recommended to undergo follow-up with gastroscopy or endoscopic ultrasonography at 3,6 , and 12 months postoperatively. If there was no residual tumor or recurrence, a gastroscopic reexamination was performed annually thereafter. If the pathological examination indicated a GIST, an enhanced chest and abdominal CT or MRI was necessary to evaluate tumor recurrence or distant metastasis.

\section{Statistical analysis}


All data were analyzed through descriptive statistics. Measurement data are expressed as mean \pm standard deviation or median (range). Enumeration data are described using frequencies and percentages. The statistical analysis of all data was performed using SPSS version 22.0 (IBM).

\section{Results}

1.

Baseline characteristics

A total of 21 patients ( 21 lesions) were enrolled in the study. Table 1 shows the patients' baseline characteristics. The male-to-female ratio was $13: 8$, and the mean age was $44.2 \pm 14.4$ (range, 19-65) years. The most common tumor location was the cardia, with $14(66.7 \%)$ cases. Otherwise, there were 3 $(14.3 \%)$ cases of tumors in the lower esophagus and $2(9.5 \%)$ cases each of tumors in the upper and middle esophagus. The mean tumor size was $38.2 \pm 12.2$ (range, 12-60) $\mathrm{mm}$; most of the tumors were $35-50 \mathrm{~mm}$ in diameter (15 cases; $71.4 \%)$. There were $5(23.8 \%)$ cases of tumors $<3.5 \mathrm{~cm}$ and only 1 $(4.8 \%)$ case of a tumor $>50 \mathrm{~mm}$. All patients underwent preoperative endoscopic ultrasonography; the outcomes showed that all SMTs originated from the esophageal MP, primarily the superficial MP (15 cases $[71.4 \%]$ ). There were $6(28.6 \%)$ cases of tumors in close contact with the deep MP. 
Table 1

Baseline patient characteristics

\begin{tabular}{|ll|}
\hline & $\mathrm{N}(\%)$ \\
\hline No. of patients & 21 \\
\hline No. of lesions & 21 \\
\hline Sex, $\mathrm{n}(\%)$ & \\
\hline Male & $13(61.9)$ \\
\hline Female & $8(38.1)$ \\
\hline Age, yr(range) & $44.2 \pm 14.4(19-65)$ \\
\hline Tumor location & \\
\hline Upper esophagus & $2(9.5)$ \\
\hline Middle esophagus & $2(9.5)$ \\
\hline Lower esophagus & $3(14.3)$ \\
\hline Cardia & $14(66.7)$ \\
\hline Tumor size, mm(range) & $38.2 \pm 12.2(12-60)$ \\
\hline No. of tumors of different sizes, $\mathrm{n}(\%)$ & \\
\hline$\Phi \unrhd 35$ mm & $5(23.8)$ \\
\hline $35 m m \leq \Phi \leq 50$ mm & $15(71.4)$ \\
\hline$\Phi \unrhd 50$ mm & $1(4.8)$ \\
\hline Tumor origin & $15(71.4)$ \\
\hline Superficial MP & $6(28.6)$ \\
\hline Deep MP & \\
\hline
\end{tabular}

2.

Clinical and pathological outcome

The O-STER operation was successful in all patients (Table 2). The mean operation time was $56.7 \pm 19.3$ (range, 20-112) minutes. All tumors were successfully resected, and the en bloc and complete resection rates were both $100 \%$. With respect to operation-related complications, there was only $1(4.8 \%)$ case of postoperative subcutaneous emphysema of the neck. In this case, the tumor was in the upper esophagus and had a diameter of $50 \mathrm{~mm}$. Furthermore, the tumor was closely related to the muscular layer since it originated from the deep MP layer. Therefore, the tumor was difficult to dissect; the muscular layer was damaged and a tiny perforation was observed. However, the subcutaneous emphysema rapidly and 
spontaneously resolved with the use of metal clips to close the tunnel openings, and no delayed perforation or gastrointestinal fistula formation occurred postoperatively. The mean hospital length of stay was $5.2 \pm 2.0$ (range, $3-10$ ) days. Based on the pathological findings, leiomyomas were detected in $18(85.7 \%)$ cases and GISTs were detected in only $3(14.3 \%)$ cases; the GISTs were classified as having a very low or low risk according to the NIH 2008 modified GIST risk classification criteria. The pathological results also confirmed negative margins in all resected tumors. All patients underwent follow-up for a mean $26.7 \pm 16.9$ months postoperatively. During the follow-up period, no cases of residue, recurrence, or distant metastases were observed, and there were no cases of delayed bleeding, perforation, or other serious complications. 
Table 2

Clinical and pathological outcomes of patients treated with O-STER

\begin{tabular}{|ll|}
\hline & Overall \\
\hline Operation time, min(range) & $56.7 \pm 19.3(20-112)$ \\
\hline En bloc resection, $\mathrm{n}(\%)$ & $21(100)$ \\
\hline Complete resection, $\mathrm{n}(\%)$ & $21(100)$ \\
\hline Complication, $\mathrm{n}(\%)$ & \\
\hline Pneumothorax & $0(0)$ \\
\hline Subcutaneous emphysema & $1(4.8)$ \\
\hline Pneumoperitoneum & $0(0)$ \\
\hline Delayed bleeding & $0(0)$ \\
\hline Delayed perforation & $0(0)$ \\
\hline Hospitalization time, days(range) & $5.2 \pm 2.0(3-10)$ \\
\hline Pathological results, $\mathrm{n}(\%)$ & \\
\hline Leiomyoma & $18(85.7)$ \\
\hline GIST, low risk & $2(9.5)$ \\
\hline GIST, very low risk & $1(4.8)$ \\
\hline Specimen margin, $\mathrm{n}(\%)$ & $0(0)$ \\
\hline positive & $21(100)$ \\
\hline negative & $0(0)$ \\
\hline Follow-up time, months(range) & \\
\hline Follow-up outcomes, $\mathrm{n}(\%)$ & \\
\hline Residue & \\
\hline Recurrence & \\
\hline Metastasis & \\
\hline
\end{tabular}

\section{Discussion}

Esophageal SMT is a rare disease with a low incidence in clinical practice. Leiomyoma is the most common pathological type, accounting for $70-80 \%$ of benign esophageal tumors. The middle and lower 
esophagus are the primary sites, which may be related to the muscle composition of these areas of the esophagus [11, 12]. GIST is another type of esophageal SMT, with an incidence of $<5 \%$. GISTs are believed to arise from the interstitial cells of Cajal [13]. Other rare types include granular cell tumors and lipomas. Although most esophageal SMTs are benign, GISTs tend to become malignant, and patients with tumors $>5 \mathrm{~cm}$ may develop clinical symptoms such as dysphagia or substernal pain owing to tumor location and size, thus affecting quality of life. Therefore, timely and complete tumor resection is essential.

Surgery has long been considered the standard of care for gastrointestinal SMTs; however, it is invasive and associated with many complications. With the continuous improvement of endoscopic techniques, ESD has gradually been used to treat SMTs of the esophagus and cardia. Studies have confirmed that ESD is minimally traumatic and associated with lower complication rates. ESD also has a shorter operation time, making it a preferable treatment option. However, for SMTs derived from the MP, the complete resection rate of ESD is relatively low. Bialek et al. [5] reported that the complete resection rate of SMTs originating from the MP using ESD was only $68.2 \%$. Lee et al. [6] confirmed that the complete resection rate of ESD was 75\%. Chinese professors have proposed ESE, EFTR, and other procedures related to ESD that are widely used to treat upper gastrointestinal SMTs. Studies have shown that the complete resection rate of ESE has significantly improved, exceeding $90 \%$; however, the rates of bleeding and perforation are also relatively high. Liu et al. [14] reported that the complete resection rate of ESE was $96.8 \%$; however, the perforation rate reached $12.9 \%$. Ye et al. [7] demonstrated that the complete resection rate of ESE was $95.6 \%$, whereas the perforation rate was $8.9 \%$. EFTR is safe and effective for the treatment of gastric SMTs, and studies have proven that its complete resection rate can reach up to 87.5-100\% $[15,16]$, making it suitable for gastrointestinal SMTs arising from the deep MP and growing into the lumen. Although the technique has been proven safe and effective for gastric lesions, the esophageal wall is thinner than the stomach wall and has only an outer membrane (i.e. it lacks a serous membrane), which may lead to serious complications such as perforation, secondary intra-abdominal infection, and gastrointestinal fistula formation. Therefore, its application in esophageal SMTs is relatively limited.

Xu et al. first reported the use of STER, which is based on ESD and POEM, for the treatment of upper gastrointestinal SMTs. In 2012, our center also successfully applied this technique to treat upper gastrointestinal SMTs [17]. Subsequently, many researchers in China and other countries have attempted to apply STER for the resection of esophageal SMTs. Ye et al. [18] conducted a prospective study in 85 patients with SMTs originating from the upper gastrointestinal MP treated with STER, confirming its safety and efficacy. Wang et al. [19] reported the successful resection of SMTs originating from the upper gastrointestinal MP in 83 patients with STER, verifying its safety, feasibility, and efficacy. In 2018, we also retrospectively analyzed 128 cases of esophageal SMTs treated with STER at 4 centers and demonstrated the safety and efficacy of STER for esophageal SMTs [20].

Many studies have shown that STER is a safe and effective treatment for the resection of esophageal SMTs; however, we found over the course of the study that for some special esophageal SMTs, such as 
those located in the upper esophagus or cardia or those $>3.5 \mathrm{~cm}$ in size, due to the limited space in the tunnel and the operative difficulty, the operation time would be longer and the complication rates of STER would greatly increase. In 2015, Jun et al. [21] first reported successful opening of the mucosa on the distal end of the tunnel during STER to resect a 40-mm esophageal SMT. In 2017, Zhang et al. [22] reported 10 cases of SMTs near the cardia that were successfully resected using submucosal tunneling endoscopic resection with double opening (DO-STER), which demonstrated that DO-STER is safe and effective for removing SMTs located within the cardia. Therefore, we made technical improvements to STER and named the new technique O-STER. By constructing a second tunnel opening, the pressure inside the tunnel is reduced, the operating space in the tunnel is increased, and the field of view of marginal tumor dissection is improved, making the resection of the aforementioned types of SMTs safer and more convenient.

The key to the STER technique is the successful construction of a submucosal tunnel. The operating space within the tunnel greatly affects its successful dissociation and reduces the occurrence of complications. However, the upper esophagus and the cardia are two special locations that are associated with increased STER difficulty. For SMTs located in the upper esophagus, tunnel construction is difficult due to the short distance from the esophageal entrance. An insufficient tunnel length results in insufficient operating space within the tunnel, making SMT dissection more difficult. SMTs in the cardia border the stomach, resulting in insufficient tunnel space. In addition, a limited field of view at the junction increases the occurrence of complications. Therefore, for the dissection of SMTs in such cases, increasing the operating space and expanding the field of view are essential. The O-STER procedure allows for expansion of the operating space and field of view of the tunnel via the distal end opening of the tunnel, making SMT resection easier and reducing the duration of operation and risk of complications. In this study, 2 (9.5\%) tumors were in the upper esophagus versus $14(66.7 \%)$ in the cardia. They were all completely resected during uneventful operations without complications. No residual tumor or recurrence was noted during the follow-up period.

The appropriate SMT size for removal by STER is controversial. Most professors believe that STER is suitable for the resection of SMTs $\leq 3.5 \mathrm{~cm}$; however, some studies suggest that STER can also be effective for the resection of SMTs $>3.5 \mathrm{~cm}$. Ye et al. [18] reported that STER is an effective and safe treatment for upper gastrointestinal SMTs $\leq 3 \mathrm{~cm}$; however, they stated that the operation is too challenging for SMTs $>3 \mathrm{~cm}$. Wang et al. [19] believed that STER is safe and effective for SMTs $>3.5 \mathrm{~cm}$. The Chinese consensus on SMT diagnosis and management in 2018 [23] proposed that STER could be used to remove SMTs $\leq 5 \mathrm{~cm}$. In previous studies, we also confirmed that STER is safe and effective for resecting SMTs $\leq 5 \mathrm{~cm}$. We also found that using STER to resect esophageal SMTs $\leq 3.5 \mathrm{~cm}$ is easier and requires a short operation time; conversely, for the resection of SMTs $>3.5 \mathrm{~cm}$, insufficient operating space in the tunnel increases the operative difficulty, the operation duration, and complication rates and obviously reduces the complete resection rate.

Li et al. [24] used STER procedures to resect 47 SMTs at the gastroesophageal junction, among which complete resection was achieved in only 35 cases (74.5\%) and the complication rate reached $8.6 \%$. They 
found that tumor diameter $\geq 2 \mathrm{~cm}$ and irregular shape were the main factors responsible for STER failure. Du et al. [25] used STER for 89 cases of esophageal SMTs and found that the complete resection rate was only $78.7 \%$ and the complication rate reached $21.3 \%$. They confirmed that SMT size is a key factor in the piecemeal resection of STER procedures and that the resection of SMTs $>3.5 \mathrm{~cm}$ is very difficult. Song et al. [26] performed STER in 10 cases of SMTs $>3.5 \mathrm{~cm}$ and demonstrated a mean operation duration of 156 (range $60-320$ ) minutes and a complete resection rate of only $80 \%$ and perforation rate of $10 \%$, thus confirming that STER is difficult for SMTs $>3.5 \mathrm{~cm}$. Chen et al. [27] also confirmed that a transverse diameter $>3.5 \mathrm{~cm}$ was a significant risk factor for segmental resection of STER, the occurrence of complications, and technical difficulty. In the present study, 16 (76.2\%) of the enrolled patients had SMTs > $3.5 \mathrm{~cm}$; all were successfully resected by O-STER. The operation time was significantly shorter and there were no serious complications, indicating that O-STER may be a safer and more effective treatment for SMTs $>3.5 \mathrm{~cm}$.

In this study, 21 lesions were successfully resected by O-STER. The mean operation duration was 56.7 minutes, which is consistent with that reported for STER (40-78.3 minutes). All SMTs were preoperatively evaluated by endoscopic ultrasonography. Although common endoscopy can provide a preliminary understanding of lesion size and morphology, the diagnostic sensitivity and specificity for SMTs are only $87 \%$ and $29 \%$, respectively [28]. Endoscopic ultrasonography is the most accurate endoscopic method for evaluating SMTs in the gastrointestinal tract, which can evaluate SMT size, origin, growth pattern, and internal properties. In the present study, the en bloc and complete resection rates were both $100 \%$ and there was only 1 (4.8\%) case of subcutaneous emphysema during the operation; no complications occurred postoperatively. According to the literature, the complete resection rate of SMTs by STER can reach $80-100 \%$; however, the perforation rate is $3-10 \%$, and the gas-related complication rate is $2.4-$ $12.1 \%[25,26,29,30]$. The resection and complication rates of O-STER were similar to those of STER. Postoperative pathological findings showed that most SMTs were classified as leiomyomas (85.7\%) and only 3 cases were classified as GISTs; however, all were considered low or very low risk. All specimens had negative vertical and horizontal margins. During the mean follow-up period of 26.7 months, no residue, recurrence, metastasis, or other serious complications were observed.

In summary, the 0-STER technique has the following advantages: (1) reduces pressure inside the tunnel, increasing the operating space, especially for SMTs $>3.5 \mathrm{~cm}$, and shortening the operation time; (2) allows a good field of view for SMTs located in the upper esophagus and cardia through opening of the distal tunnel and expanding of the tunnel space; (3) increases the space inside the tunnel owing to a second tunnel opening, thereby reducing the operation-related difficulty, rates of bleeding and perforation in the tunnel, operation time, and complication rates.

\section{Conclusions}

O-STER is safe and effective for the treatment of special SMTs located within the upper esophagus and cardia or those with a diameter $>3.5 \mathrm{~cm}$. It can significantly increase the operating space and reduce the occurrence of complications. However, only a few cases from a single center were included in the present 
study. Moreover, this study had a retrospective design. In future studies, large-scale multi-center data must be included to validate our findings.

\section{Abbreviations}

SMTs

Esophageal submucosal tumors;

MP

Muscularis propria;

ESD

Endoscopic submucosal dissection;

ESE

Endoscopic submucosal excavation;

EFTR

endoscopic full-thickness resection;

POEM

Peroral endoscopic myotomy;

STER

Submucosal tunneling endoscopic resection;

O-STER

Open submucosal tunneling endoscopic resection;

ECG

Electrocardiography;

PPIs

Proton pump inhibitors;

GIST

Gastrointestinal stromal tumor;

DO-STER

Submucosal tunneling endoscopic resection with double opening.

\section{Declarations}

\section{Ethics approval and consent to participate}

This study was approved by the Ethics Committee of Shenzhen Hospital of Southern Medical University. Written informed consent was obtained from patients.

\section{Consent for publication}

Not applicable. 


\section{Competing interests}

Authors declare that they have no competing interests.

\section{Acknowledgments}

Silin Huang and Sufang Tu contributed equally to this work as first authors. We must acknowledge all authors for their support and contributions.

\section{Funding}

The study was supported by the Clinical Research Startup Program of Southern Medical University by High-level University Construction Funding of Guangdong Provincial Department of Education (LC2016PY008).

\section{Authors' contributions}

Study concepts: Silin Huang, Wei Gong. Study design: Silin Huang, Wei Gong. Data acquisition: Sufang Tu, Jingwen Fu, Genhua Yang, Xiaowei Tang. Data analysis and interpretation: Silin Huang, Sufang Tu, Jingwen Fu, Xiaowei Tang. Manuscript preparation: Sufang Tu. Manuscript editing: Silin Huang, Sufang Tu. Manuscript review: Wei Gong. All authors have read and approved the manuscript.

\section{Availability of data and materials}

The datasets used and/or analyzed during the current study are available from the corresponding author on reasonable request.

\section{References}

1. Codipilly DC, Fang H, Alexander JA, Katzka DA, Ravi K. Subepithelial esophageal tumors: a singlecenter review of resected and surveilled lesions. GASTROINTEST ENDOSC. 2018;87:370-7.

2. Ioannis SPKT. Endoscopic ultrasonography for gastric submucosal lesions. World.

3. J Gastrointest Endosc 2011;3:86-94.

4. Mutrie CJ, Donahue DM, Wain JC, et al. Esophageal Leiomyoma: A 40-Year Experience. The Annals of Thoracic Surgery. 2005;79:1122-5. 
5. Seremetis MG, Lyons WS, DeGuzman VC, Peabody JJ. Leiomyomata of the esophagus. An analysis of 838 cases. CANCER-AM CANCER SOC. 1976;38:2166-77.

6. Bialek A, Wiechowska-Kozlowska A, Pertkiewicz J, et al. Endoscopic submucosal dissection for treatment of gastric subepithelial tumors (with video). GASTROINTEST ENDOSC. 2012;75:276-86.

7. Lee I, Lin PY, Tung S, et al. Endoscopic submucosal dissection for the treatment of intraluminal gastric subepithelial tumors originating from the muscularis propria layer. ENDOSCOPY. 2006;38:1024.

8. Ye LP, Zhu LH, Zhou XB, Mao XL, Zhang Y. Endoscopic excavation for the treatment of small esophageal subepithelial tumors originating from the muscularis propria. Hepatogastroenterology. 2015;62:65-8.

9. Inoue $\mathrm{H}$, Minami H, Kobayashi $\mathrm{Y}$, et al. Peroral endoscopic myotomy (POEM) for esophageal achalasia. ENDOSCOPY. 2010;42:265-71.

10. Xu MD, Cai MY, Zhou PH, et al. Submucosal tunneling endoscopic resection: a new technique for treating upper $\mathrm{Gl}$ submucosal tumors originating from the muscularis propria layer (with videos). GASTROINTEST ENDOSC. 2012;75:195-9.

11. Inoue $\mathrm{H}$, Ikeda $\mathrm{H}$, Hosoya $\mathrm{T}$, et al. Submucosal endoscopic tumor resection for subepithelial tumors in the esophagus and cardia. ENDOSCOPY. 2012;44:225.

12. Ko WJ, Song GW, Cho JY. Evaluation and Endoscopic Management of Esophageal Submucosal Tumor. Clin Endosc. 2017;50:250-3.

13. Humphris JL, Jones DB. Subepithelial mass lesions in the upper gastrointestinal tract. J GASTROEN HEPATOL. 2008;23:556-66.

14. Kang G, Kang Y, Kim KH, et al. Gastrointestinal stromal tumours of the oesophagus: a clinicopathological and molecular analysis of 27 cases. HISTOPATHOLOGY. 2017;71:805-12.

15. Liu B, Song J, Qu B, et al. Endoscopic muscularis dissection for upper gastrointestinal subepithelial tumors originating from the muscularis propria. Surg Endosc. 2012;26:3141-8.

16. Tan $Y$, Tang $X$, Guo $T$, et al. Comparison between submucosal tunneling endoscopic resection and endoscopic full-thickness resection for gastric stromal tumors originating from the muscularis propria layer. Surg Endosc. 2017;31:3376-82.

17. Zhou PH, Yao LQ, Qin XY, et al. Endoscopic full-thickness resection without laparoscopic assistance for gastric submucosal tumors originated from the muscularis propria. SURG ENDOSC. 2011;25:2926-31.

18. Gong W, Xiong Y, Zhi F, et al. Preliminary experience of endoscopic submucosal tunnel dissection for upper gastrointestinal submucosal tumors. ENDOSCOPY. 2012;44:231-5.

19. Ye L, Zhang Y, Mao X, et al. Submucosal tunneling endoscopic resection for small upper gastrointestinal subepithelial tumors originating from the muscularis propria layer. Surg Endosc. 2014;28:524-30. 
20. Wang H, Tan Y, Zhou Y, et al. Submucosal tunneling endoscopic resection for upper gastrointestinal submucosal tumors originating from the muscularis propria layer. EUR J GASTROEN HEPAT. 2015;27:776-80.

21. Tu S, Huang S, Li G, et al. Submucosal Tunnel Endoscopic Resection for Esophageal Submucosal Tumors: A Multicenter Study. Gastroenterol Res Pract. 2018;2018:2149564.

22. Ng JJ, Chiu PWY, Shabbir A, So JBY. Removal of a large, 40-mm, submucosal leiomyoma using submucosal tunneling endoscopic resection and extraction of specimen using a distal mucosal incision. ENDOSCOPY 2015;47 Suppl 1 UCTN:E232.

23. Zhang $Q$, Cai J, Xiang $L$, et al. Modified submucosal tunneling endoscopic resection for submucosal tumors in the esophagus and gastric fundus near the cardia. ENDOSCOPY. 2017;49:784.

24. Zhou P, Zhong Y, Li Q. [Chinese Consensus on Endoscopic Diagnosis and Management of Gastrointestinal Submucosal Tumor(Version 2018)]. Zhonghua Wei Chang Wai Ke Za Zhi. 2018;21:841-52.

25. Li Z, Gao Y, Chai N, et al. Effect of submucosal tunneling endoscopic resection for submucosal tumors at esophagogastric junction and risk factors for failure of en bloc resection. Surg Endosc. 2018;32:1326-35.

26. Du C, Ma L, Chai N, et al. Factors affecting the effectiveness and safety of submucosal tunneling endoscopic resection for esophageal submucosal tumors originating from the muscularis propria layer. Surg Endosc. 2018;32:1255-64.

27. Song S, Feng M, Zhou H, Liu M, Sun M. Submucosal Tunneling Endoscopic Resection for Large and Irregular Submucosal Tumors Originating from Muscularis Propria Layer in Upper Gastrointestinal Tract. J Laparoendosc Adv Surg Tech A. 2018;28:1364-70.

28. Chen T, Lin ZW, Zhang YQ, et al. Submucosal Tunneling Endoscopic Resection vs Thoracoscopic Enucleation for Large Submucosal Tumors in the Esophagus and the Esophagogastric Junction. J Am Coll Surg. 2017;225:806-16.

29. Rosch T, Kapfer B, Will U, et al. Accuracy of endoscopic ultrasonography in upper gastrointestinal submucosal lesions: a prospective multicenter study. Scand J Gastroenterol. 2002;37:856-62.

30. Li Q, Chen W, Zhang C, et al. Clinical impact of submucosal tunneling endoscopic resection for the treatment of gastric submucosal tumors originating from the muscularis propria layer (with video). Surg Endosc. 2015;29:3640-6.

31. Xiong $\mathrm{Y}, \mathrm{Hu} \mathrm{H}$, Wang A, et al. [Preliminary experience with endoscopic gastric submucosal tumor resection through the submucosal tunnel using double tunnel and double flex endoscope]. Nan Fang Yi Ke Da Xue Xue Bao. 2015;35:455-8.

\section{Figures}




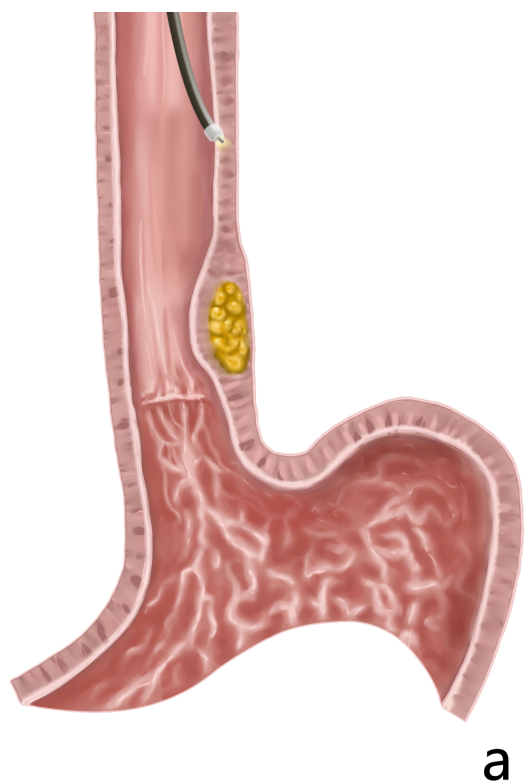

a

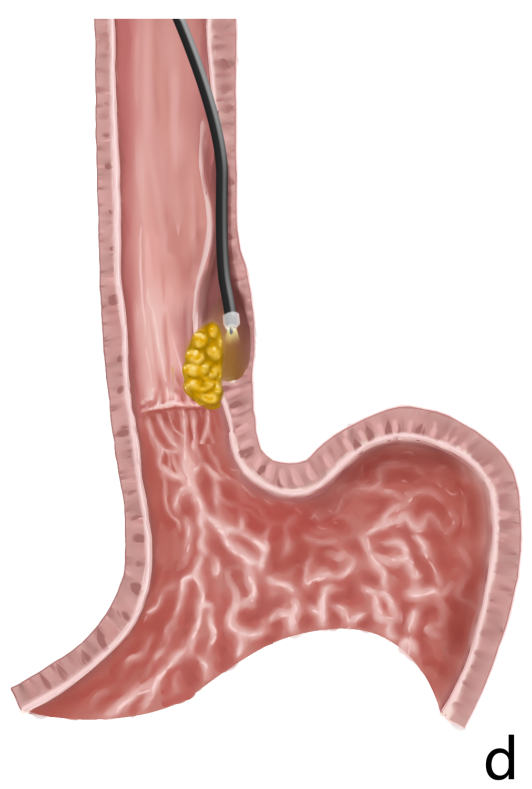

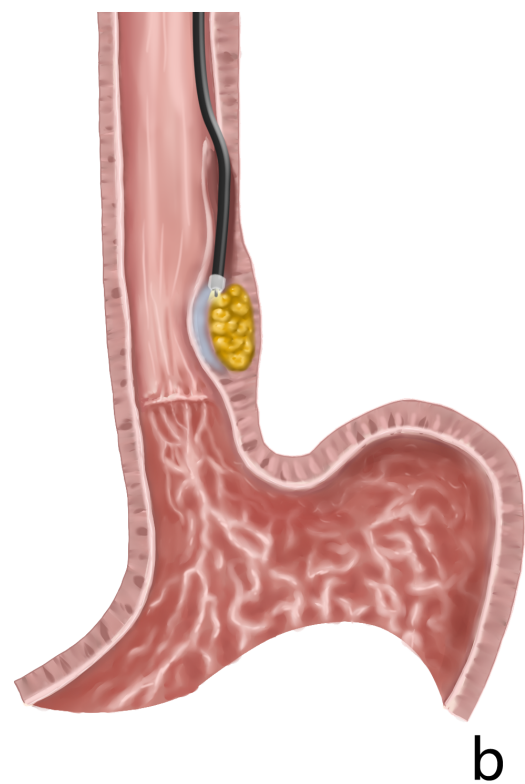

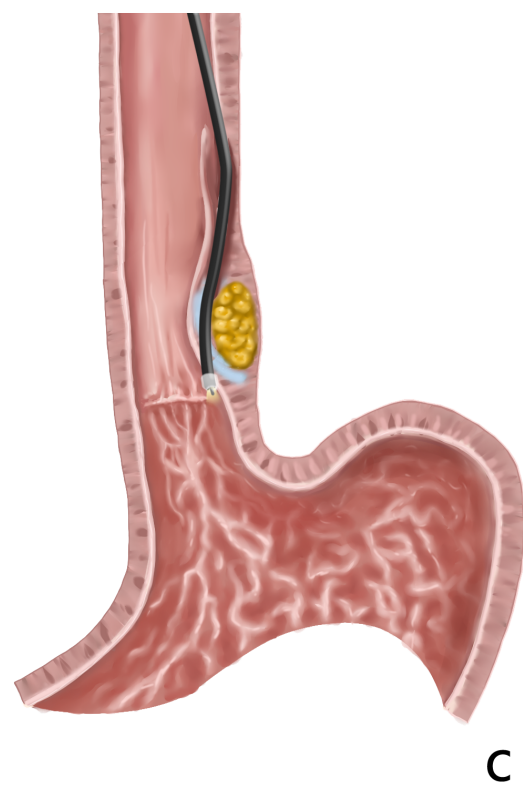

\section{Figure 1}

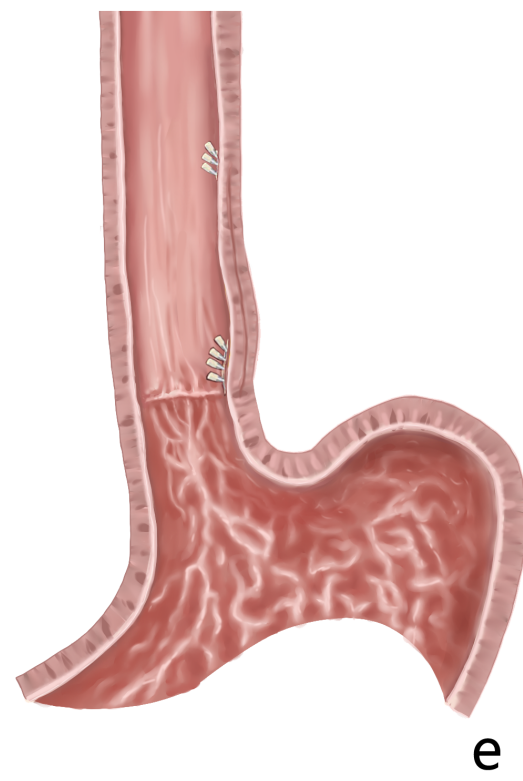

Key procedures of O-STER for esophageal SMT. (a) A SMT located at the middle esophagus; mucosal incision and creation of the tunnel entrance. (b) Creation of the submucosal tunnel. (c) Creating the second tunnel opening. (d) Dissection of the SMT gradually and pushing the tumor out of the second tunnel. (e) Closing the two tunnel openings. 

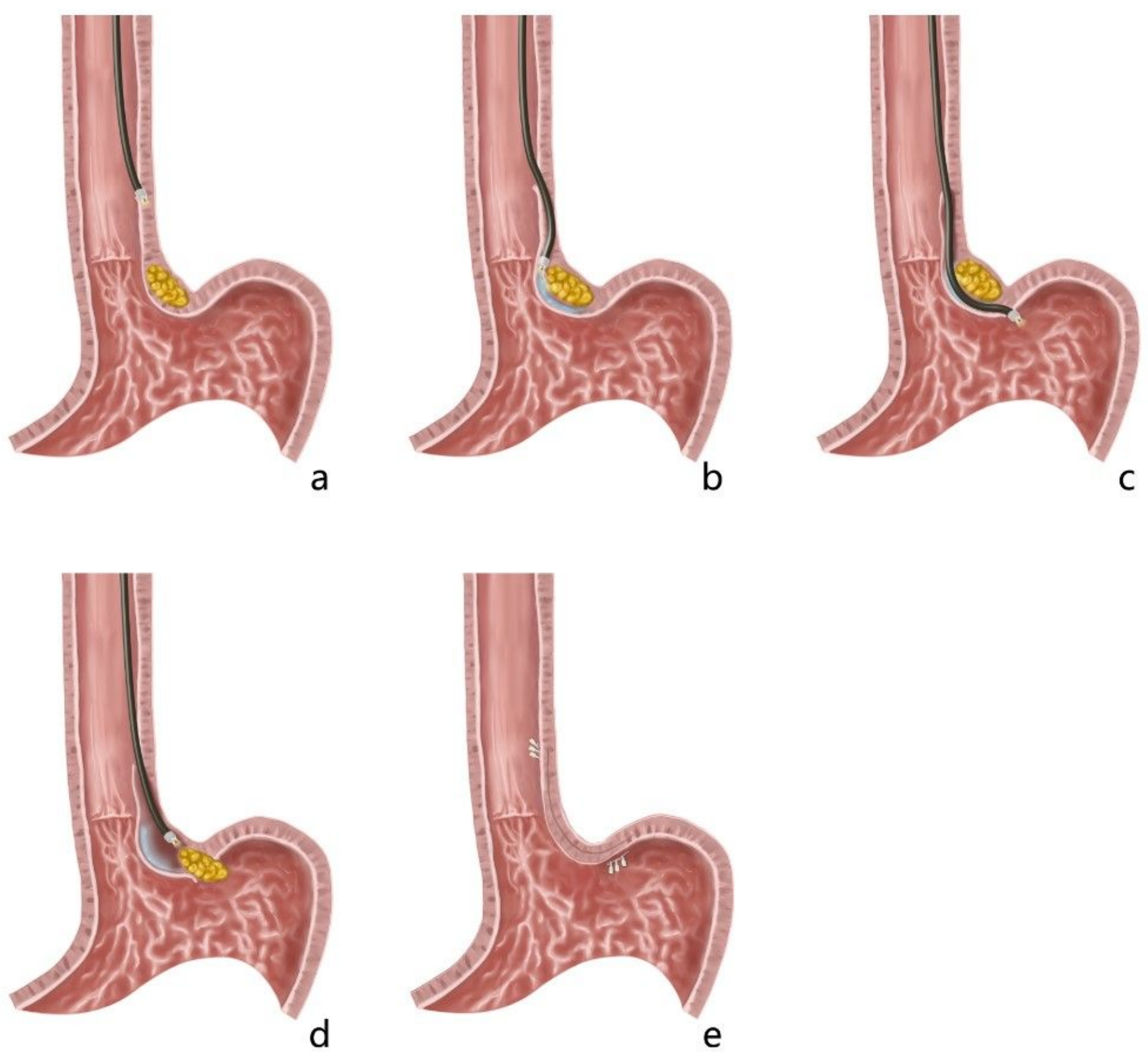

Figure 2

Key procedures of O-STER for cardial SMT. (a) A SMT located in the cardia and making longitudinal mucosal incision to create the tunnel entrance. (b) constructing a straight submucosal tunnel from the oral to the anal side (c) Creating the second tunnel opening and dissection of the tumor. (d) Pushing the tumor out of the second tunnel. (e) Closure of the tunnel. 

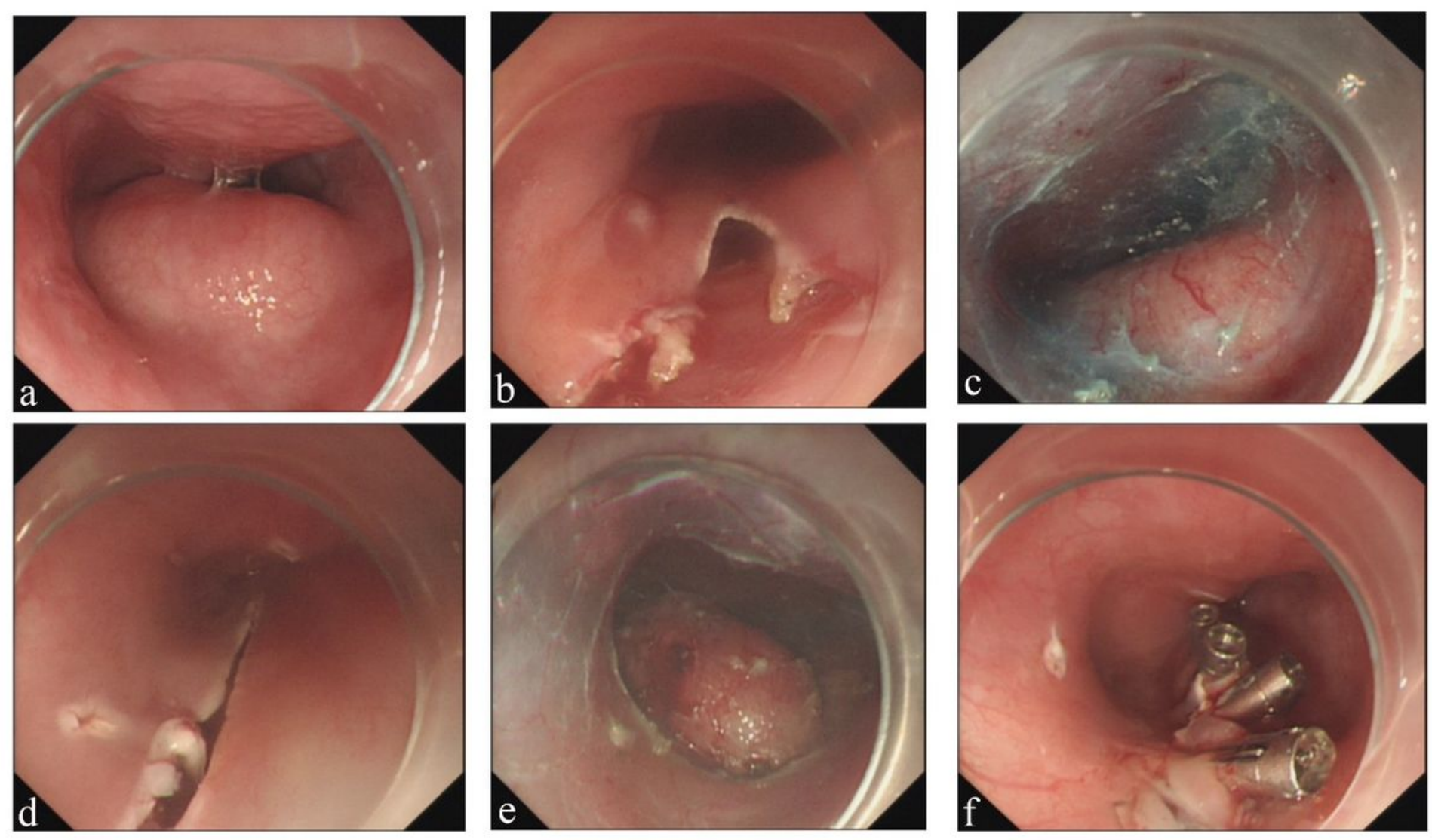

\section{Figure 3}

Case illustration of O-STER for a middle esophageal SMT. (a) A SMT located in the middle esophagus. (b) Mucosal incision. (c) Creating the first tunnel and dissecting the tumor. (d) Creating the second tunnel opening. (e) Dissecting the tumor gradually and pushing the tumor out of the second tunnel. (f) Removal of the tumor and closure of the two tunnel openings. 

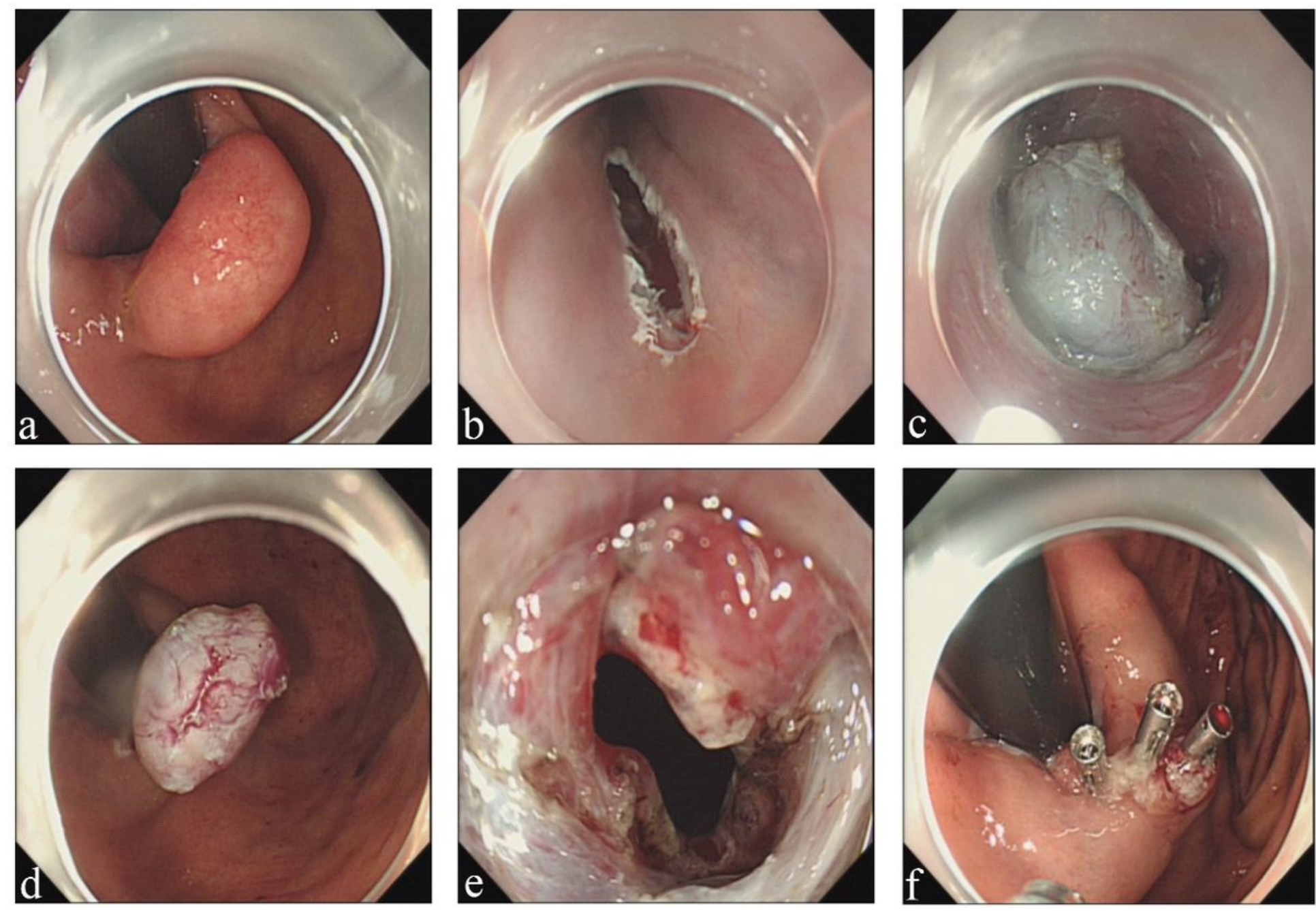

\section{Figure 4}

Case illustration of O-STER for cardial SMT. (a) A SMT located at the cardia. (b) Mucosal incision. (c) Creating the first tunnel and dissecting the tumor. (d) Creating the second tunnel. (e) Pushing the tumor out of the second tunnel. (f) Tumor resection finished and the tunnel closure. 